TITLE:

\title{
Formation of thermal vacancies on the Si sublattice of the intermetallic compound MoSi2
}

\section{$\operatorname{AUTHOR}(\mathrm{S}):$}

Zhang, XY; Sprengel, W; Staab, TEM; Inui, H; Schaefer, HE

\section{CITATION:}

Zhang, XY ...[et al]. Formation of thermal vacancies on the Si sublattice of the intermetallic compound MoSi2. Physical Review Letters 2004, 92(15): 155502.

ISSUE DATE:

2004-04-16

URL:

http://hdl.handle.net/2433/50079

RIGHT:

Copyright 2004 American Physical Society 


\title{
Formation of Thermal Vacancies on the Si Sublattice of the Intermetallic Compound $\mathrm{MoSi}_{2}$
}

\author{
X. Y. Zhang, ${ }^{1,2, *}$ W. Sprengel, ${ }^{2}$ T. E. M. Staab, ${ }^{3}$ H. Inui, ${ }^{4}$ and H.-E. Schaefer ${ }^{2}$ \\ ${ }^{1}$ Key Laboratory of Metastable Materials Science and Technology, Yanshan University, 066004 Qinhuangdao, China \\ ${ }^{2}$ Institut für Theoretische und Angewandte Physik, Universität Stuttgart, D-70550 Stuttgart, Germany \\ ${ }^{3}$ HISKP, Bonn University, Nuss-Allee 14-16, D-53115 Bonn, Germany \\ ${ }^{4}$ Department of Materials Science and Engineering, Kyoto University, Kyoto 606-8501, Japan
}

(Received 22 July 2003; published 14 April 2004)

\begin{abstract}
For a detailed understanding of high-temperature processes in complex solids the identification of the sublattice on which thermal defects are formed is of basic interest. Theoretical studies in intermetallic compounds favor a particular sublattice for thermal vacancy formation. In the present study we detect in ordered $\mathrm{MoSi}_{2}$ thermal vacancies with a low formation enthalpy of $H_{V}^{F}=(1.6 \pm 0.1) \mathrm{eV}$, and we succeed in showing by experimental and theoretical efforts that they are preferentially formed on the $\mathrm{Si}$ sublattice. By these data self-diffusion in $\mathrm{MoSi}_{2}$ can be understood.
\end{abstract}

DOI: 10.1103/PhysRevLett.92.155502

PACS numbers: 61.72.Ji, 61.72.Cc, 66.30.Lw

The binary ordered intermetallic compound $\mathrm{MoSi}_{2}$ [see Fig. 1(a)] is a potential material for high-temperature structural applications [1]. The high-temperature properties [2-4] of intermetallic compounds are mainly determined by thermal defects such as vacancies. A study of the thermodynamic properties of thermally formed vacancies in $\mathrm{MoSi}_{2}$ and particularly of the determination of the sublattice of the compound on which these thermal vacancies are formed is of particular interest for the understanding of these properties. The formation of thermal vacancies has been predicted on the transition-metal sublattice in some intermetallic compounds [5,6]. Recent positron lifetime and dilatometry experiments [7-10] have contributed to the understanding of thermal vacancy formation and migration in intermetallic compounds. These techniques are, however, incapable of determining the sublattice of an intermetallic compound on which the thermal vacancies are formed so that more specific techniques are desirable. Coincident measurements of the Doppler broadening of the positron-electron annihilation photons have been successfully employed in order to identify the location of radiation-induced vacancies $[11,12]$. By this technique the atomic chemical environment of the annihilation site, e.g., a vacancy has been analyzed by considering the high-momentum part of the electron momentum distribution [13-16]. In the present Letter we demonstrate that Doppler broadening measurements can be successfully employed in the exemplary case of $\mathrm{MoSi}_{2}$ in order to identify the sublattices on which high-temperature vacancies are formed. This method might also be of general and wide interest for the studies of thermal atomic defects in complex solids.

For the present high-temperature positron annihilation studies, a positron source of ${ }^{58} \mathrm{CoCl}$ with an activity of $1.2 \times 10^{6} \mathrm{~Bq}$ was deposited inside the borehole of a cylinder prepared from a $\mathrm{MoSi}_{2}$ single crystal [17], annealed in a hydrogen atmosphere, closed by a $\mathrm{MoSi}_{2}$ cover, and sealed in a quartz tube under a pressure of $p<10^{-3} \mathrm{~Pa}$.
The positron annihilation lifetime spectra (time resolution $260 \mathrm{ps}, 10^{6}$ coincidence counts) were analyzed by means of standard techniques [7]. The coincident measurements of the Doppler broadening of the annihilation photons with energies $E_{1}$ and $E_{2}$ were carried out by a collinear setup of two high-resolution Ge detectors with an energy resolution $\Delta E=1.2 \mathrm{keV}$. The high peak-tobackground ratio $\left(>5 \times 10^{5}\right)$ was achieved by diagonal cuts of the $\left(E_{1}, E_{2}\right)$-Doppler spectra along the $E_{1}+E_{2}=$ $(1022 \pm 1) \mathrm{keV}$ energy conservation line $[13,18]$. With the spectra of $>10^{7}$ coincidence counts we achieved good statistics in the region $E \geq(511+5) \mathrm{keV}$ corresponding to $p_{L} \geq\left(19.6 \times 10^{-3}\right) m_{0} c$ for the momenta of the chemically characteristic core electrons $\left(m_{0}\right.$ is electron rest mass and $c$ is the velocity of light). The reference spectra of pure Mo and Si at ambient temperature were measured in a sandwich arrangement with a ${ }^{22} \mathrm{NaCl}$ positron source. The $W$ parameter characterizes

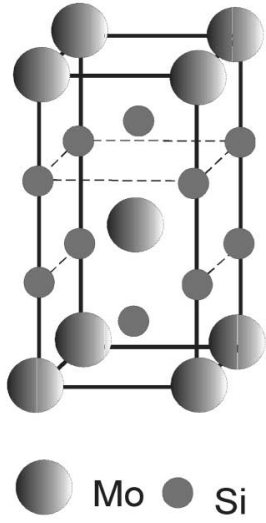

(a)

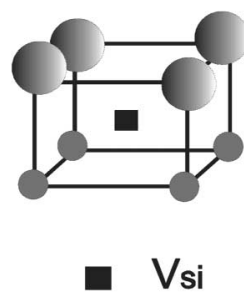

(b)

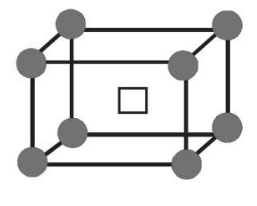

VMo

(c)
FIG. 1. Perfect and defected lattice structures of $\mathrm{MoSi}_{2}$ : (a) defect-free $\mathrm{MoSi}_{2}$; (b) a vacancy located on the $\mathrm{Si}$ sublattice; (c) a vacancy located on the Mo sublattice. 
the core-electron fractional contribution to the Doppler broadening spectra in the range of $\pm(20-28) \times 10^{-3} m_{0} c$.

Theoretical studies of the positron annihilation Doppler broadening parameters were performed by using the wave functions of free atoms [19,20]. The total electron momentum distribution was obtained by summing up the contributions from each core-electron state weighted with its partial annihilation rate calculated by employing the generalized gradient approximation (GGA) of the electron-positron correlation [21].

The positron lifetime of $\tau=(117 \pm 1)$ ps determined for $\mathrm{MoSi}_{2}$ at ambient temperature, which is confirmed by GGA calculations for delocalized positrons, demonstrates that structural vacancies in $\mathrm{MoSi}_{2}$ at ambient temperature are negligible. This is concluded from the comparison of the positron lifetime $\tau$ and the valence electron density of $\mathrm{MoSi}_{2}, \rho_{\mathrm{el}}=338 \mathrm{~nm}^{-3}$, with the corresponding values for pure metals and intermetallics [12].

For temperatures below $1050 \mathrm{~K}$ the free positron lifetime shows a weak temperature dependence (see Fig. 2) $\tau_{f}(T)=\tau_{f_{0}}(1+\alpha T)$ with $\tau_{f_{0}}=117 \mathrm{ps}$, the free positron lifetime at room temperature, and a thermal expansion coefficient $\alpha=5 \times 10^{-5} \mathrm{~K}^{-1}$, which is similar to the volume expansion coefficient of $\beta=2.58 \times 10^{-5} \mathrm{~K}^{-1}$ [22]. A reversible S-shaped increase of the mean positron lifetime $\bar{\tau}$ is observed for $T>1050 \mathrm{~K}$. This is attributed to positron trapping at thermally formed vacancies. The analysis of the positron lifetime spectra can be described by one type of thermal vacancy with a positron lifetime $\tau_{1}=(165 \pm 5) \mathrm{ps}$.

For the two-state positron trapping model (see Ref. [7]), the positron trapping rate $\sigma C_{V}$ of thermally formed vacancies is given by

$$
\sigma C_{V}(T)=\frac{\bar{\tau}-\tau_{f}}{\tau_{f}\left(\tau_{1}-\bar{\tau}\right)}=I_{1}\left(\frac{1}{\tau_{0}}-\frac{1}{\tau_{1}}\right),
$$

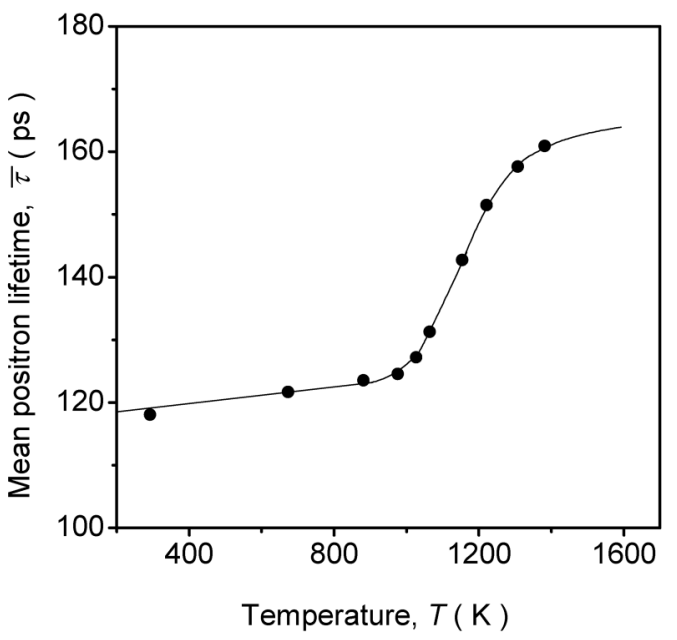

FIG. 2. Thermal formation of vacancies is indicated by the reversible $S$-shaped temperature variation of the mean positron lifetime $\bar{\tau}$. The solid line is a fit of Eq. (3) to the experimental data. with the vacancy concentration

$$
C_{V}(T)=\exp \left(\frac{S_{V}^{F}}{k_{B}}\right) \exp \left(-\frac{H_{V}^{F}}{k_{B} T}\right) .
$$

Here, $\sigma$ is the temperature-independent specific positron trapping rate, $\tau_{f}$ is the positron lifetime in the defect-free crystal, $\bar{\tau}$ is the mean positron lifetime, $H_{V}^{F}$ and $S_{V}^{F}$ are, respectively, the enthalpy and the entropy of vacancy formation, and $k_{B}$ is Boltzmann's constant. The time constants $\tau_{0}=\left[\left(1 / \tau_{f}\right)+\sigma C_{V}\right]^{-1}$ and $\tau_{1}$ (characteristic for vacancies) as well as the relative intensities $I_{1}$ and $I_{0}=1-I_{1}$ can be deduced from the analyses of the positron lifetime spectra.

A fit of the temperature variation of the mean positron lifetime

$$
\bar{\tau}(T)=\tau_{f} \frac{1+\sigma \exp \left(S_{V}^{F} / k_{B}\right) \exp \left(-H_{V}^{F} / k_{B} T\right) \tau_{1}}{1+\sigma \exp \left(S_{V}^{F} / k_{B}\right) \exp \left(-H_{V}^{F} / k_{B} T\right) \tau_{f}}
$$

derived from Eqs. (1) and (2) to the data in Fig. 2 taking into account the linear thermal expression $\tau_{f}(T)$ mentioned above, yields $H_{V}^{F}=(1.6 \pm 0.1) \mathrm{eV}$ and $\sigma \exp \left(S_{V}^{F} / k_{B}\right)=(4.0 \pm 0.5) \times 10^{12} \mathrm{~s}^{-1}$.

When a positron is trapped at a lattice vacancy the ratio of the annihilation probabilities with core or valence electrons is reduced compared to that of a positron delocalized on interstices as demonstrated by studies of vacancies in pure $\mathrm{Si}$ [23] and in pure Al [24]. Therefore, the temperature dependence of the Doppler broadening $W$ parameter (see Fig. 4) obtained from the high-momentum part of the electron momentum distribution of $\mathrm{MoSi}_{2}$ (see Fig. 3) shows an S-shaped behavior in analogy to the positron lifetime measurements (Fig. 2) according to

$$
W(T)=W_{f} \frac{1+\sigma C_{V} W_{1}}{1+\sigma C_{V} W_{f}},
$$

where $W_{f}$ and $W_{1}$ denote the $W$ parameters characteristic for the defect-free compound and for saturation trapping

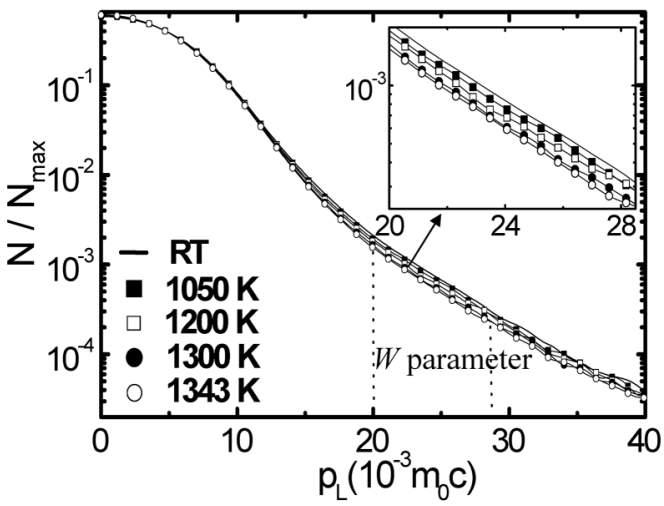

FIG. 3. Coincident Doppler broadening spectra measured for $\mathrm{MoSi}_{2}$ at high temperatures. The spectra are normalized to the total number of counts. The high momentum range representing positron annihilation with core electrons as indicated is characterized by the $W$ parameter. 


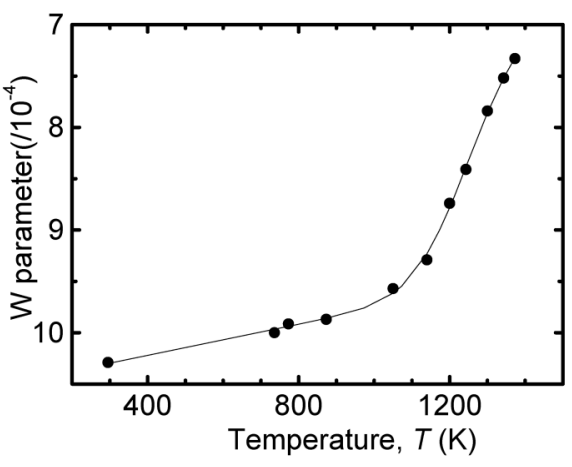

FIG. 4. Temperature variation of the $W$ parameter in $\mathrm{MoSi}_{2}$. The solid line is a fit to the data according to Eq. (4) (note the reversed ordinate scale).

of positrons at thermal vacancies, respectively. A fit of Eq. (4) to the data in Fig. 4 yields the value of the vacancy formation enthalpy $H_{V}^{F}=(1.7 \pm 0.1) \mathrm{eV}$ similar to the value derived from the positron lifetime studies.

Normalizing the vacancy formation enthalpy found in $\mathrm{MoSi}_{2}$ to the melting temperature $T_{M}$ of the $\mathrm{MoSi}_{2}$ yields a relatively low value $H_{V}^{F} / k_{B} T_{M}=8.0$ similar to the low values $H_{V}^{F} / k_{B} T_{M}=7.0$ to 9.5 reported for openstructured bcc-type intermetallic compounds [7,25] with high concentrations of thermal vacancies. This directly demonstrates the formation of high concentrations of thermal vacancies in $\mathrm{MoSi}_{2}$.

For identifying the sublattice of $\mathrm{MoSi}_{2}$ on which thermal vacancies are formed, measurements of the coincident Doppler broadening at 1343 K (see Fig. 5) are employed. At this temperature the majority of the positrons is annihilated in thermally formed vacancies according to the intensity $I_{1}=0.8$ derived from the positron lifetime measurements.

It turns out that the Doppler broadening data measured on $\mathrm{MoSi}_{2}$ at $1343 \mathrm{~K}$ can be modeled at high electron momenta much better by the theoretical data obtained for vacancies $V_{\mathrm{Si}}$ on the Si sublattice (see Fig. 5) than by the theoretical data for $V_{\mathrm{Mo}}$. This demonstrates that thermal vacancies at high temperatures in $\mathrm{MoSi}_{2}$ are preferentially formed on the $\mathrm{Si}$ sublattice, whereas vacancies $V_{\text {Mo }}$ on the Mo sublattice are negligible within the experimental uncertainty limits. If any contribution of $V_{\mathrm{Mo}}$ would be taken into account by a combination of the theoretical curves in Fig. 5 this would lower the theoretical values and thereby deteriorate the fit to the experimental data.

A preferential formation of vacancies on the Si sublattice in $\mathrm{MoSi}_{2}$ is also anticipated from the purely experimental data in Fig. 6. The high-temperature data for $\mathrm{MoSi}_{2}$ exhibit the nonzero high-momentum slope typical for Mo atoms (curve $b$ in Fig. 6) which are available as nearest neighbors only for vacancies on the Si sublattice [see Fig. 1(b)].

The present studies of high-temperature vacancy formation can be directly employed for an immediate

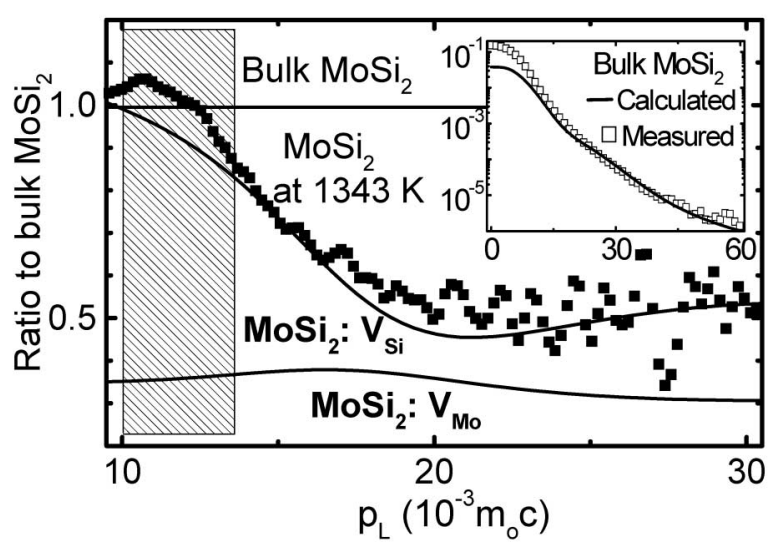

FIG. 5. Ratio curves of the high-momentum part of the electron momentum distribution for a $\mathrm{MoSi}_{2}$ single crystal at $1343 \mathrm{~K}(\boldsymbol{\square})$ and the calculated data for the vacancies $V_{\mathrm{Si}}$ and $V_{\text {Mo }}$ on the two sublattices of $\mathrm{MoSi}_{2}$. The experimental and theoretical data for vacancy trapping were normalized to the experimental and theoretical data of defect-free bulk $\mathrm{MoSi}_{2}$ (see the inset) at ambient temperature. No atomic relaxation around the vacancies is considered. The experimental data for $\mathrm{MoSi}_{2}$ at $1343 \mathrm{~K}$ are scaled to full trapping at thermal vacancies by making use of the intensity $I_{1}=0.8$ derived from the positron lifetime measurements, which yields a fraction of trapped positrons $\eta=0.54$. The theoretical results are not valid at low momenta (shaded area) due to the omission of the valence electron contribution in the calculations. The calculated ratio curve of the high-momentum part for vacancies on the $\mathrm{Si}$ sublattice $\left(V_{\mathrm{Si}}\right)$ is in good agreement with experimental data of the $\mathrm{MoSi}_{2}$ at $1343 \mathrm{~K}$, demonstrating that thermal vacancies are formed preferentially on the Si sublattice of the $\mathrm{MoSi}_{2}$.

understanding of recent self-diffusion studies of ${ }^{31} \mathrm{Si}$ [26] and of ${ }^{99} \mathrm{Mo}$ [27] in $\mathrm{MoSi}_{2}$. The diffusion of $\mathrm{Si}$ in $\mathrm{MoSi}_{2}$ is demonstrated to be faster than that of Mo by more than 6 orders of magnitude, and an activation enthalpy $Q^{\mathrm{SD}}=(2.3 \pm 0.1) \mathrm{eV}$ is determined. From a simple approach for the diffusion activation enthalpy, $Q=H_{V}^{F}+H_{V}^{M}$, a rather low value for the vacancy migration enthalpy of $H_{V}^{M}=0.7 \mathrm{eV}$ for $\mathrm{MoSi}_{2}$ can be anticipated taking into account the above value of $H_{V}^{F}=$ $1.6 \mathrm{eV}$. This indicates a high mobility of thermal vacancies on the $\mathrm{Si}$ sublattice of $\mathrm{MoSi}_{2}$. The high atomic diffusivity on the majority $\mathrm{Si}$ sublattice of $\mathrm{MoSi}_{2}$ [26] can therefore be explained by a high vacancy concentration on the majority sublattice in combination with a high vacancy mobility or a low vacancy migration enthalpy.

A low value of the vacancy migration enthalpy $H_{V}^{M}$ in $\mathrm{MoSi}_{2}$ is confirmed by considering the temperature dependence of the equilibration time of the thermal vacancy concentration [7]. This has been estimated from the shift of the first moments of the positron lifetime spectra after fast cooling from 1380 to 1073 or to $873 \mathrm{~K}$. The mean positron lifetime characteristic for the lower temperatures was reached within less than $10 \mathrm{~min}$, yielding an estimate of $H_{V}^{M}<1.1 \mathrm{eV}$ by assuming a temperature 


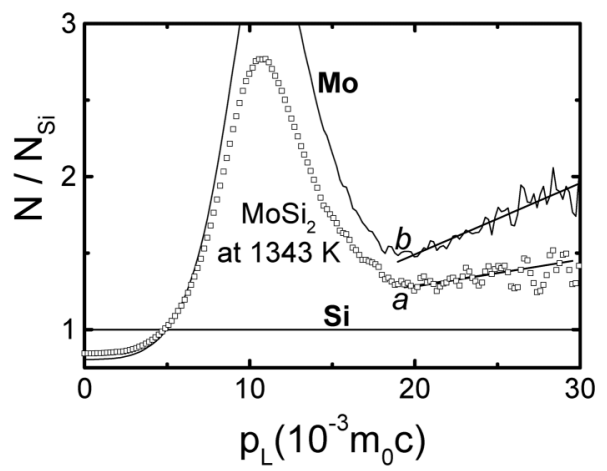

FIG. 6. Ratio curves of the coincidently measured Doppler broadening spectra of pure $\mathrm{Mo}$ and of pure $\mathrm{Si}$ at ambient temperature as well as that of $\mathrm{MoSi}_{2}$ at $1343 \mathrm{~K}$. All spectra are normalized to the spectrum of defect-free pure $\mathrm{Si}$. The slopes $a$ for $\mathrm{MoSi}_{2}$ at $1343 \mathrm{~K}$ and $b$ for the pure Mo are determined by fitting the data in the range of $[(20-28) \times$ $\left.10^{-3}\right] m_{0} c$. The value for slope $a$ lies between slope $b$ of pure Mo and the horizontal line of pure Si indicating a Mo contribution as well as a $\mathrm{Si}$ contribution. Thus, the vacancy environment contains Mo atoms indicating the formation of thermal vacancies on the Si sublattice (see $V_{\mathrm{Si}}$ in Fig. 1).

dependence of the time constant $t_{\mathrm{E}}$ for thermal vacancy equilibration of $t_{\mathrm{E}}^{-1}=t_{\mathrm{E}, 0}{ }^{-1} \exp \left[-H_{V}^{M} /\left(k_{B} T\right)\right]$ and taking a value for $t_{\mathrm{E}, 0}-1=4 \times 10^{5} \mathrm{~s}^{-1}$ similar to the values for other intermetallic compounds as B2-FeAl [10].

In conclusion, the present studies on the ordered intermetallic compound $\mathrm{MoSi}_{2}$ demonstrate that, in addition to the detection of thermally formed vacancies by positron lifetime spectroscopy, the sublattice can be experimentally identified on which these vacancies are formed. This is of importance for understanding hightemperature solid state processes on an atomic level and of wide interest for understanding, e.g., diffusion mechanisms, order-disorder processes, solid state reactions, etc.

X.Y.Z. thanks the AvH Foundation for support. We are indebted to the DFG for financial support. The help of $\mathrm{K}$. Blaurock and K. J. Reichle in the experiments as well as of $\mathrm{H}$. Wendel in specimen preparation is acknowledged.

*Corresponding author.

Electronic address: xyzh66@yahoo.com.cn

[1] K. Ito, T. Yano, T. Nakamoto, M. Moriwaki, H. Inui, and M. Yamaguchi, Prog. Mater. Sci. 42, 193 (1997).

[2] Diffusion Mechanisms in Crystalline Materials, edited by Y. Mishin, G. Vogl, N. E. B. Cowern, C. R. A. Catlow, and D. Farkas, MRS Symposia Proceedings No. 527 (Materials Research Society, Pittsburgh, 1998).

[3] O. Dimitrov, in Intermetallic Compounds-Principles and Practice, edited by J. H. Westbrook and R. L. Fleischer (John Wiley, Chichester, 1994), Vol. 1, p. 771.
[4] G. Sauthoff, in Diffusion in Ordered Alloys, edited by B. Fultz, R.W. Cahn, and D. Gupta (TMS, Warrendale, 1993), p. 205.

[5] C. L. Fu, Y. Y. Ye, M. H. Yoo, and K. M. Ho, Phys. Rev. B 48, 6712 (1993).

[6] M. Fähnle, J. Mayer, and B. Meyer, Intermetallics 7, 315 (1999).

[7] R. Würschum, C. Grupp, and H.-E. Schaefer, Phys. Rev. Lett. 75, 97 (1995).

[8] J. Wolff, A. Broska, M. Franz, B. Köhler, and Th. Hehenkamp, Mater. Sci. Forum 255-257, 593 (1997).

[9] A. H. van Ommen and J. de Miranda, Philos. Mag. A 43, 387 (1981).

[10] H.-E. Schaefer, K. Frenner, and R. Würschum, Phys. Rev. Lett. 82, 948 (1999).

[11] A. A. Rempel, K. Blaurock, K. J. Reichle, W. Sprengel, J. Major, and H.-E. Schaefer, Phys. Rev. Lett. 89, 185501 (2002).

[12] X. Y. Zhang, W. Sprengel, K. Blaurock, A. A. Rempel, K. J. Reichle, K. Reimann, H. Inui, and H.-E. Schaefer, Phys. Rev. B 66, 144105 (2002).

[13] P. A. Kumar, M. Alatalo, V. J. Ghosh, A. C. Kruseman, B. Nielsen, and K. G. Lynn, Phys. Rev. Lett. 77, 2097 (1996).

[14] K. Saarinen, T. Laine, K. Skog, J. Mäkinen, and P. Hautojärvi, K. Rakennus, P. Uusimaa, A. Salokatve, and M. Pessa, Phys. Rev. Lett. 77, 3407 (1996).

[15] U. Myler, R. D. Goldberg, A. P. Knights, D. W. Lawther, and P. J. Simpson, Appl. Phys. Lett. 69, 3333 (1996).

[16] K. Saarinen, J. Nissilä, H. Kauppinen, M. Hakala, M. J. Puska, P. Hautojärvi, and C. Corbel, Phys. Rev. Lett. 82, 1883 (1999).

[17] H. Inui, K. Ishikawa, and M. Yamaguchi, Intermetallics 8, 1131 (2000).

[18] K. G. Lynn and A. N. Goland, Solid State Commun. 18, 1549 (1976).

[19] M. Alatalo, H. Kauppinen, K. Saarinen, M. J. Puska, J. Mäkinen, P. Hautojärvi, and R. M. Nieminen, Phys. Rev. B 51, 4176 (1995).

[20] M. Alatalo, B. Barbiellini, M. Hakala, H. Kauppinen, T. Korhonen, M. J. Puska, K. Saarinen, P. Hautojärvi, and R. M. Nieminen, Phys. Rev. B 54, 2397 (1996).

[21] B. Barbiellini, M. J. Puska, T. Korhonen, A. Harju, T. Torsti, and R. M. Nieminen, Phys. Rev. B 53, 16201 (1996).

[22] O. Thomas, J. P. Senateur, P. Madar, O. Laborde, and E. Rosencher, Solid State Commun. 55, 629 (1985).

[23] S. Szpala, P. Asoka-Kumar, B. Nielsen, J. P. Peng, S. Hayakawa, K. G. Lynn, and H.-J. Gossmann, Phys. Rev. B 54, 4722 (1996).

[24] K. G. Lynn, J. E. Dickman, W. L. Brown, and M. F. Robbins, Phys. Rev. B 20, 3566 (1979).

[25] H.-E. Schaefer, K. Frenner, and R. Würschum, Intermetallics 7, 277 (1999).

[26] M. Salamon, A. Strohm, T. Voss, P. Laitinen, I. Riihimäki, S. Divinski, W. Frank, J. Räisänen, and H. Mehrer, Philos. Mag. 84, 737 (2004).

[27] M. Salamon and H. Mehrer, Defect Diffus. Forum 216217, 161 (2003). 